\title{
Vorwort der Herausgeberin
}

Kommt der „Bibliotheksdienst“ nicht zur Ruhe? Anfang 2013 der Wechsel von Herstellung und Vertrieb von der Zentral- und Landesbibliothek Berlin hin zum Verlag De Gruyter. Dann zur Jahresmitte die gemeinsame Herausgebertätigkeit von Zentral- und Landesbibliothek Berlin und Landesbibliothekszentrum RheinlandPfalz, nun eine neue Umschlagsgestaltung mit den Corporate-Design-Farben des nun alleinigen Herausgebers, dem Landesbibliothekszentrum Rheinland-Pfalz ...

Die Zentral- und Landesbibliothek Berlin muss Prioritäten setzen. Sie plant den zukünftigen Neubau am Berliner Tempelhofer Feld und die „Welt der Sprachen“ im Humboldt-Forum. Das fordert allen Einsatz, steht doch nicht weniger zur Debatte als die Neukonzeption einer Metropolenbibliothek.

Die Zentral- und Landesbibliothek Berlin hatte 2002 nach Auflösung des ehemaligen Deutschen Bibliotheksinstituts den „Bibliotheksdienst“ übernommen und viele Jahre allein alle Herausforderungen und alle Lasten getragen. Durch die Abgabe von Herstellung und Vertrieb an einen renommierten Verlag war die Möglichkeit zur Weiterentwicklung der Zeitschrift gegeben, die gemeinsame Herausgeberschaft mit dem Landesbibliothekszentrum Rheinland-Pfalz war eine willkommene Unterstützung. Nun wird dieser Schritt konsequent fortgesetzt und das Landesbibliothekszentrum Rheinland-Pfalz übernimmt ab 2014 allein die herausgeberische Verantwortung für die inhaltliche Weiterentwicklung.

Gerne möchten wir die Gelegenheit nutzen, das Landesbibliothekszentrum Rheinland-Pfalz ${ }^{1}$ den Leserinnen und Lesern näher vorzustellen. Es entstand 2004 durch Fusion der „Pfälzischen Landesbibliothek“ in Speyer (gegründet 1921), der „Rheinischen Landesbibliothek“ in Koblenz (gegründet 1987), der „Bibliotheca Bipontina“ in Zweibrücken (geht zurück auf historische Bestände aus dem 16. Jahrhundert), der Büchereistelle in Neustadt a.d. Weinstraße (gegründet 1921) und der Büchereistelle in Koblenz (gegründet 1948). Was wie eine Verwaltungseinheit klingt, ermöglicht viel mehr. Das LBZ schafft eine Struktur für das rheinland-pfälzische Bibliothekswesen. Es arbeitet spartenübergreifend für öffentliche und wissenschaftliche Bibliotheken als „Dienstleistungs- und Kompetenzzentrum“, wie es der „Errichtungserlass“ formuliert. Somit steht das LBZ mitten in allen Fragen, die Bibliotheken heute umtreiben müssen. Als Bibliothek mit landesbibliothekarischer Funktion ist es mit dem Sammeln von Literatur aus und über die Region betraut und daher für die Region zuständig. Dies zeigt sich z. B. an Angeboten wie LITexpress (dem rheinland-pfälzischen Lieferdienst von Büchern und Medien), edoweb (Archivserver für elektronische Dokumente und

1 Siehe auch www.lbz-rlp.de. 
landeskundliche Websites) oder dilibri (dem rheinland-pfälzischen Digitalisierungsportal). Leseförderung ist eine der zentralen Aufgaben in einem Bundesland, das sich politisch vor allem den Fragen der Bildung verschrieben hat. Daher sind die Büchereistellen bekannt durch „Lesesommer“ und „Bücherminis“, „Geschichtenkoffer" und Basiskurse für die vielen ehrenamtlichen Bibliothekare (in Rheinland-Pfalz liegt der Anteil ehrenamtlicher öffentlicher Bibliotheken bei über $80 \%$ ).

Aufgrund dieses vielseitigen und anspruchsvollen Aufgabenkatalogs ist das LBZ in der idealen Position, eine Zeitschrift wie den Bibliotheksdienst, der sowohl das öffentliche wie auch das wissenschaftliche Bibliothekswesen in seiner gesamten Breite abdeckt, herauszugeben. Viele Leserinnen und Leser werden auch andere Publikationen des LBZ kennen, so beispielsweise die rheinland-pfälzische Bibliothekszeitschrift „Bibliotheken heute“. Widmet sich diese Zeitschrift den regionalen Themen und Projekten, ist der „Bibliothekdienst“ als überregionale und allgemein bibliothekarische Fachzeitschrift eine spannende Herausforderung und sinnvolle Ergänzung.

In der Aufgabe der Herausgabe des Bibliotheksdienstes steht das Landesbibliothekszentrum Rheinland-Pfalz nicht allein. Mit dem Beirat für den Bibliotheksdienst, der 2013 berufen wurde, stehen engagierte Vertreterinnen und Vertreter des öffentlichen und wissenschaftlichen Bibliothekswesens mit Rat und ihren Netzwerken zur Verfügung, um spannende Themen für die Beiträge der Zeitschrift aufzugreifen und anzuregen. Für die Landesbibliotheken freuen wir uns, dass Julia Freifrau Hiller von Gaertringen, die die Badische Landesbibliothek Karlsruhe leitet und derzeit Vorsitzende der AG Regionalbibliotheken im Deutschen Bibliotheksverband ist, als Vertreterin der Landesbibliotheken gewonnen werden konnte. Lars Jendral (ebenfalls Landesbibliothekszentrum) verlässt den Beirat, da das LBZ jetzt die Aufgabe der Herausgabe der Zeitschrift hat. Ihm ist zu danken für seine engagierte Mitarbeit.

Doch eine Zeitschrift lebt nur von ihren Autorinnen und Autoren und so bedanken wir uns ausdrücklich bei allen, die im letzten Jahr interessante Aufsätze geschrieben haben. Es war der Wunsch der Herausgeber und des Beirats, das thematische Spektrum zu verbreitern, was auch schon mit ersten Ansätzen gelungen ist. So werden auch Randthemen aufgegriffen und der Bibliotheksdienst präsentiert sich zunehmend „experimentierfreudig“. Mit der regelmäßigen Kolumne der „Zukunftswerkstatt“ finden auch die ganz neuen Themen Eingang; „klassische“ Themen wie Erschließung oder Kulturguterhalt sollen ebenso wenig vernachlässigt werden. Besonders viel Anerkennung in Fachkreisen erhielten die Sonderhefte $\mathrm{zu}$ „Digitalisierung und Bestandserhaltung“ und „e-Pflichtgesetzgebung in den Bundesländern“. Kritische Debatten sind durchaus willkommen und so bitten wir um neue Beiträge. Bei uns können die Autoren zu Wort kommen, auf- 
wändige Previewingverfahren gibt es nicht und die Redaktion hält sich bewusst bei „Eingriffen“ zurück. Abwechslungsreich und lebendig soll unsere Zeitschrift sein und da brauchen wir Sie - als Leser/innen und als Autor/inn/en. Wünschen wir uns somit gemeinsam mit dem Verlag eine anregende Debatte rund um die Gegenwarts- und Zukunftsfragen unserer Bibliotheken, denn die Glaskugel, die uns zeigen würde, wohin unser Weg genau geht, die fehlt nicht nur unserer Redaktion.

Dr. Annette Gerlach

Leiterin Landesbibliothekszentrum Rheinland-Pfalz 